\title{
Nanostructures for Enhanced Light Absorption in Solar Energy Devices
}

\author{
Gustav Edman Jonsson, Hans Fredriksson, Raja Sellappan, and Dinko Chakarov
}

Department of Applied Physics, Chalmers University of Technology, 41296 Gothenburg, Sweden

Correspondence should be addressed to Gustav Edman Jonsson, gustav.jonsson@chalmers.se

Received 1 November 2010; Accepted 23 March 2011

Academic Editor: Mohamed Sabry Abdel-Mottaleb

Copyright (C) 2011 Gustav Edman Jonsson et al. This is an open access article distributed under the Creative Commons Attribution License, which permits unrestricted use, distribution, and reproduction in any medium, provided the original work is properly cited.

The fascinating optical properties of nanostructured materials find important applications in a number of solar energy utilization schemes and devices. Nanotechnology provides methods for fabrication and use of structures and systems with size corresponding to the wavelength of visible light. This opens a wealth of possibilities to explore the new, often of resonance character, phenomena observed when the object size and the electromagnetic field periodicity (light wavelength $\lambda$ ) match. Here we briefly review the effects and concepts of enhanced light absorption in nanostructures and illustrate them with specific examples from recent literature and from our studies. These include enhanced optical absorption of composite photocatalytically active $\mathrm{TiO}_{2} / \mathrm{graphitic}$ carbon films, systems with enhanced surface plasmon resonance, field-enhanced absorption in nanofabricated carbon structures with geometrical optical resonances and excitation of waveguiding modes in supported nanoparticle assembles. The case of Ag particles plasmon-mediated chemistry of $\mathrm{NO}$ on graphite surface is highlighted to illustrate the principle of plasmon-electron coupling in adsorbate systems.

\section{Motivation}

Solar radiation is the main long-term source of energy on earth. All other known energy sources are depletable (fossil), restricted (geothermal and gravitational), or currently not socially adequate (e.g., nuclear, because of the risks of proliferation). Though important, the density of solar power impinging on earth is a mere $1.4 \mathrm{~kW} / \mathrm{m}^{2}$ [1], consequently making efficient energy conversion ever so important in light harvesting devices.

Practiced solar light harvesting schemes can be divided into two basic concepts: solar thermal and solar quantum. The former transforms solar radiation to heat, the latter into electricity or chemical fuels.

Typical devices exemplifying these concepts are the solar heat collectors and photovoltaic systems extracting solar energy in the form of electricity or by inducing a chemical reaction, for instance to produce a chemical fuel such as hydrogen. The difference in the approaches and demands on the light absorber might be understood in comparison with the natural analogues of these alternatives: in the first case, these are the atmospheric and ocean processes driven by solar radiation (wind, rain, streams, etc.) and in the second the natural photosynthesis and photolytic processes [2] of converting light energy to chemical energy and storing it in the bonds of sugar in plants, algae, and bacteria.

When solar energy is purposefully converted to heat, the aim is simply to absorb as much as possible of all available radiation. In the case of quantum transformation of the photon energy, attention must be paid to the different steps in the transforming process. Therefore, the processes of initial photon absorption, generation of charge carries, their transport, separation, and attachment should be designed in a fashion to achieve a good match between the spectral distribution of the incoming light, absorption properties of the system, and the energy levels of the transition and final states.

Transition energy levels are known to vary with the size and geometry of the active structure [3], and precise structural manipulation has become possible in the wake of nanotechnology and nanoscience hereby allowing fine tuning of transition energy levels. With this stance, all 
new construction concepts may be employed to quantum transforming devices.

In this paper, we briefly review some of the novel concepts for photon capture based on the achievements of nanoscience and nanotechnology and illustrate them with specific examples from our studies.

\section{Background}

The interaction of electromagnetic radiation with matter is classically described by the Maxwell equations in combination with the wavelength-dependent complex dielectric function

$$
\widehat{\varepsilon}(\omega)=\varepsilon_{1}(\omega)+i \varepsilon_{2}(\omega)
$$

and the complex refractive index,

$$
\hat{n}(\omega)=n(\omega)(1+i \kappa(\omega)) \equiv \sqrt{\varepsilon_{1}(\omega)+i \varepsilon_{2}(\omega)} .
$$

Being a consequence of these fundamental properties, Beer's law explicitly expresses the light field intensity at a given point, along its propagation line in some medium, according to the formula:

$$
I_{2}=I_{1} \exp \left(-\int_{P_{1}}^{P_{2}} \alpha(\bar{r}) d l\right),
$$

where $I_{1}$ and $I_{2}$ denote the intensities at the points $P_{1}$ and $P_{2}$, respectively, and $\alpha(\bar{r})=\left(4 \pi / \lambda_{0}\right) n \kappa$ where $\lambda_{0}$ denotes the wavelength in vacuum and $n \kappa$ is the purely imaginary part of the refractive index at point $\bar{r}$ [4]. A variant of Beer's law is the Lambert-Beer law which may be formulated as

$$
\Delta I_{\text {ext }}=I_{0}\left(1-\mathrm{e}^{-\#\left(\sigma_{\mathrm{abs}}+\sigma_{\mathrm{sca}}\right) z}\right) .
$$

Here $\Delta I_{\text {ext }}$ denotes the intensity loss at a point $z$ due to extinction, $\sigma_{\mathrm{abs}}$ and $\sigma_{\mathrm{sca}}$ are the absorption and scattering cross sections, respectively, and \# is the number density of such sites [5]. The term cross section should in this case strictly be interpreted as a measure of probability for a scattering or an absorption event to occur albeit it has area dimensionality. A "cross section" may be attributed to atoms, molecules, or even larger particles, making the Lambert-Beer law generally applicable.

The extinction depth, sometimes referred to as penetration depth, is a quantity defined as the physical distance into a material at which the field intensity has dropped to $1 / e$ (barely $37 \%$ ) relative to the initial intensity [4]. In this case, the Lambert-Beer law states that $-\gamma_{e} z_{\text {e.d. }}=-1 \Leftrightarrow$ $z_{\text {e.d. }}=1 / \gamma_{e}$, where $z_{\text {e.d. }}$ is the extinction depth. Using terms of refractive index, we instead obtain $z_{\text {e.d }}=\lambda_{0} / 4 \pi n \kappa$. Structures showing strong optical attenuation are often denoted as optically thick. That is when the physical thickness exceeds $z_{\text {e.d. }}$.

While the behavior of the electromagnetic field, inside a medium, is adequately described as above, the affiliating excitation processes within this medium are also essential to perceive: absorption of photons occurs when the photon energy, $h v$, and momentum match those required for an energy state transition within the absorbing material. A transition strived for in solar quantum transforming devices is the electron-hole pair generation with energy sufficient to drive the envisaged process.

When devising photon capturing structures, for electronhole pair generation, a conflict arises between the profound properties of light mentioned above and the minority carrier diffusion lengths. On one hand, designing thin solar devices is a virtue, both as smaller dimensions minimize material costs and weight but also as it minimizes the probability for unwanted intrinsic electron-hole pair recombination. Electronic diffusion length is typically $40 \mu \mathrm{m}$ for Si [6], and to minimize bulk recombination processes the cell thickness must not exceed this diffusion length of minority carriers [7]. On the other hand, the Lambert-Beer law states the minimum thickness to obtain reasonable light absorption for a given material system: the typical semiconductor materials have extinction depths on the $\mu \mathrm{m}$ scale, exceeding $100 \mu \mathrm{m}$ for $\mathrm{Si}$ in the red end of the solar spectrum. The physical constraints imposed by the mismatch between the optical and electronic length scales constitute a serious challenge for the construction of effective solar devices.

The path to circumvent this dilemma is to find innovative ways of increasing the optical thickness through manipulation of the optical density and/or the optical path length while maintaining smaller physical dimensions. Several prospects of this kind are presented in the following sections.

\section{State of the Art}

It has long been recognized that the light absorption in $\mathrm{Si}$ solar cells can be enhanced by the use of well-designed optical structures. Historically, one of the first approaches to trap light inside an absorbing structure is to use wavelength-scale textured substrates [9]. These include antireflection coatings to minimize the reflection losses at the front surface as well as structures designed to help trap the light within the cell. A frequently used structure consists of pyramids or inverted pyramids anisotropically etched into the top and also often into the rear cell surface $[9,10]$.

Diffractive optical structures [11], which allow increment of the optically effective cell thickness by a factor of 4-5, are more generally applicable both as the absorbing layers can be thinner and also not necessarily single crystalline in opposition to the method described above.

In the case of dye-sensitized solar cells, light trapping has been achieved by light scattering layers of polycrystalline anatase. These layers have proved to increase the photon to electricity conversion factor by $10 \%$. In addition, the layers have shown to be active in charge carrier generation themselves [12].

Also, photonic crystals, mimicking the electronic band structures in periodic crystals by inclusion of periodic arrays of different refractive index media into an absorbing structure have been investigated as a mean to extending the optical thickness [13-15]. In a longer perspective, more exotic socalled metamaterials $[16,17]$ with optical properties not 
achievable by naturally occurring materials might contribute to this development.

In contrast to the massive, nanostructured semiconductor materials feature stronger light absorption similarly to the metallic systems. On the other hand, semiconductor quantum dots (QDs) are of particular interest for application in solar devices for a different reason. Due to momentum delocalization and relaxation of selection, rules cross sections are enhanced compared to the bulk counterpart [18]. On the basis of impact ionization, single photon generation of multiple excitons has been demonstrated in PbSe, CdSe, and $\mathrm{PbS}$ QDs, which raises the theoretical efficiency limit of the solar cells impressively [19-21]. It is noteworthy that carrier multiplication may potentially be achieved with this approach [22].

Concentration and guiding of light on the nanometer scale can be engineered with the help of surface and particle polaritons. Plasmonic structures exhibit large extinction cross sections and present a promising way of increasing the optical thickness of solar energy devices while maintaining a physically thin structure. Plasmons are electromagnetic modes formed by light-matter coupling in the respective material and geometry. This coupling requires materials (medium) with appropriate (sometimes negative) dielectric function and particle dimensions, or surface features, smaller than the wavelength of light. The collective oscillation of conduction electrons (plasmon-polariton) or lattice vibrations in polar crystals (phonon-polariton) provide the necessary conditions [4]. Other aspect of the emerging area of plasmonics is to develop devices that will replace some electric currents with plasmon waves, because plasmons can theoretically carry huge amounts of information squeezed into the nanometer-sized wires. The practical application of plasmonics concepts suffers, however, from the fact that plasmons "live" for only 10 to 100 femtoseconds (see Figure 2) before they decay radiatively into normal light waves or transform to electron-hole pairs and eventually to vibrations (heat). The big challenge is to overcome these losses in order to put the captured light energy in work or to transfer information.

Below we present several approaches, which summarize the different principles of light utilization in nanostructures (see also Figure 1). More could be found in a recent review by Atwater and Polman [23].

Nanoparticles on a thin film of a high refractive index semiconductor may couple impingent light into waveguided modes propagating in the film. In this way, the lateral extension of the semiconductor defines its optical thickness while the vertical extension defines its physical. Hence the correlation between the two interfering quantities is abolished. This process has been demonstrated to be surprisingly efficient for waveguides with thicknesses on the nanometer scale [24-26].

Whispering gallery modes, WGM, as so-called after the acoustic phenomenon at St. Paul's Cathedral in London, occur at particular resonant wavelengths of light for a given cavity size and shape. Here the light undergoes total internal reflection at the inner surface of a cavity and becomes trapped within the void (or guide) for timescales of the order of nanoseconds [27]. Due to ellipsoidal reflection even lateral extension may be miniaturized. Obviously, the increased photon path length increases the total absorption. An illustrative example of this enhanced absorption is the observation of photolysis of water-soluble components inside cloud droplets by ultraviolet/visible radiation [28].

A similar concept for achievement of light guiding is realized by placing a plasmonic metal structure on the backside of a thin semiconductor film. The structure may couple light into surface plasmon polaritons, SPPs, propagating along the metal semiconductor interface and eventually coupling with an e-h pair excitation in the semiconductor as have been shown $[29,30]$. The principle of using perpendicular directions for absorption and diffusion is the same as above.

Nanostructures may furthermore be designed to manage the electromagnetic near field; the field energy can be spatially confined to a photoactive region in a way sometimes inspired by the natural photosynthetic antenna system. Optical nanoantennae are small plasmonic particles that resonate at visible and near-IR frequencies. In a semiconductor proximity, the antenna acts as the photon absorber while the energy is coupled to e-h pair excitations within the semiconductor via the enhanced near field [31].

Approaches to realize such structures involve, for instance, carbon nanotubes [32, 33], zeolites [34], and metallic nanoparticles [35-37]. In this context, one may also mention optical rectennas, with the aim to miniaturize the successful radio wave counterpart and directly convert light into a dc current by means of a combined antenna and rectifier structure $[18,38]$.

Our final example is the utilization of the plasmonic excitations in solar devices by tuning the decay to e-h pairs (Landau damping) [37]. In such systems, the semiconductor is redundant as a generator of charge carriers; both light absorption and charge carrier generation are facilitated in the plasmonic particle. More on plasmonic decay specificity on the nanoparticle material is presented in a paper by Langhammer et al. [39].

\section{Illustrative Examples}

Our primary interest lies in the enhanced light harvesting in photovoltaic and photocatalytic devices in which the solar light is quantum converted in photochemical or photoelectric processes by the absorbing system. The conversion of solar energy into electricity usually occurs either directly or through thermal conversion. However, it is worth noting that a new mechanism, photon-enhanced thermionic emission, which combines electric as well as thermal conversion mechanisms, is now shown to lead to enhanced conversion efficiencies that potentially could even exceed the theoretical limits of conventional photovoltaic cells [40].

4.1. Optically Active Metal (Plasmonic) Nanoparticles. As articulated above, localized surface plasmon resonances (LSPRs) are collective oscillations of the conduction electrons, which may result in optical absorption cross sections that exceed the geometric cross section by several orders of 


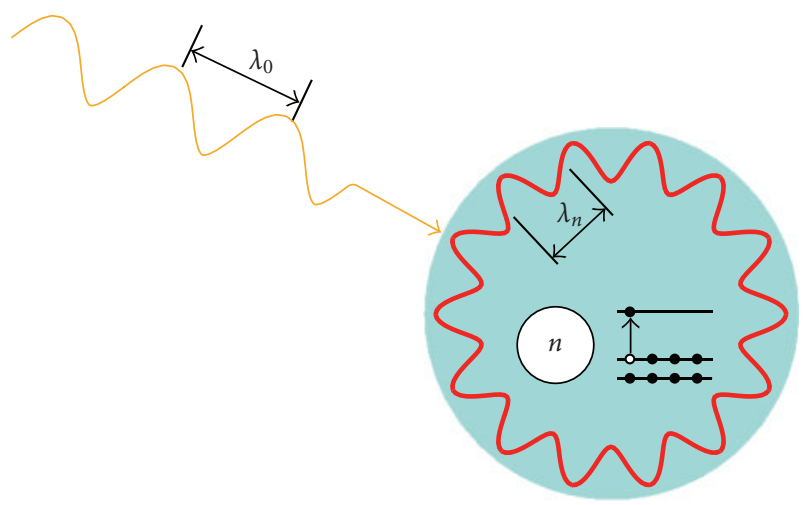

(a)

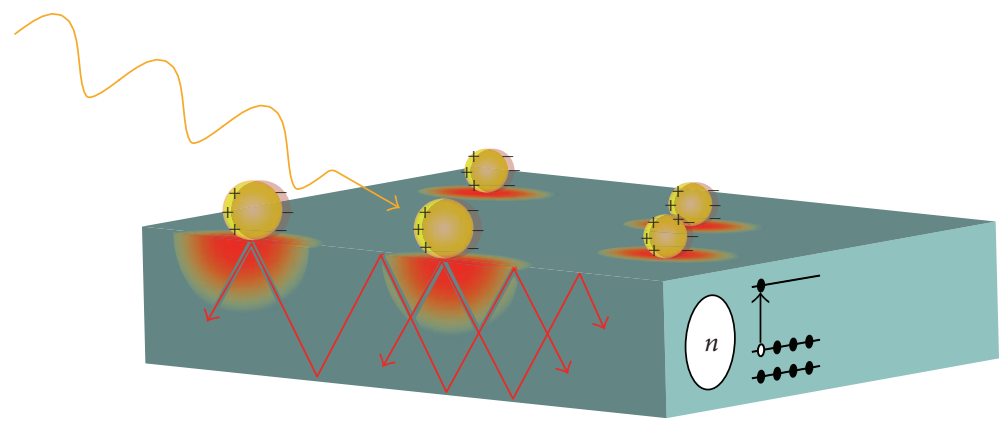

(b)

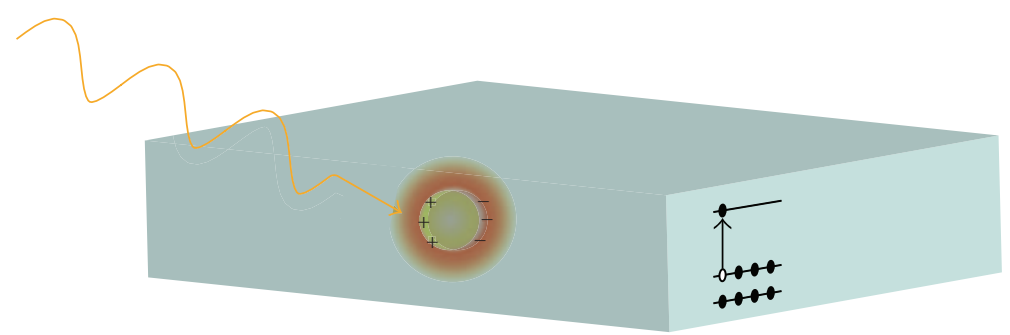

(c)

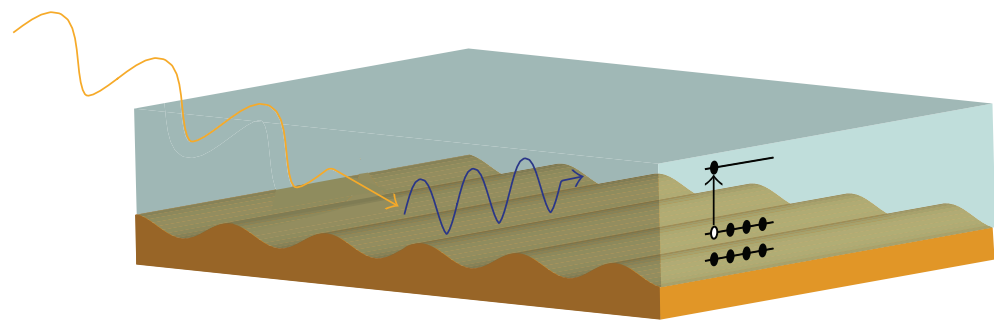

(d)

FIGURE 1: Different coupling modes of light in nanostructures and main mechanisms for charge carrier generation following the particle generation. (a) The principle of geometrical optical resonances (WGM) (note the high diffractive index). (b) Plasmon nanoparticles scatter and efficiently couple light into waveguided modes. The prolonged optical paths in (a, b) lead to enhanced total absorption and enhanced e-h-pair generation. (c) The near field intensity and gradients lead to locally increased e-h generation rates. (d) A plasmonic grating at the backside of a semiconductor couples light into SPPs that excite e-h pairs in the semiconductor with direct injection of the carriers to the empty electron bands.

magnitude. The plasmons decay either radiatively (giving rise to dramatic electromagnetic field enhancements, as used for instance, in surface-enhanced Raman spectroscopy) or into (quasi) particles such as electron-hole (e-h) pairs. See Figure 2. For many metals, the resonance wavelength falls into the near ultraviolet, visible and near infrared regime for nanostructure sizes covering the range of 20 $200 \mathrm{~nm}$. What is typical for the mentioned spectral range is that it covers (most of) the energetics of important chemical transformations, for example, bond breaking and 


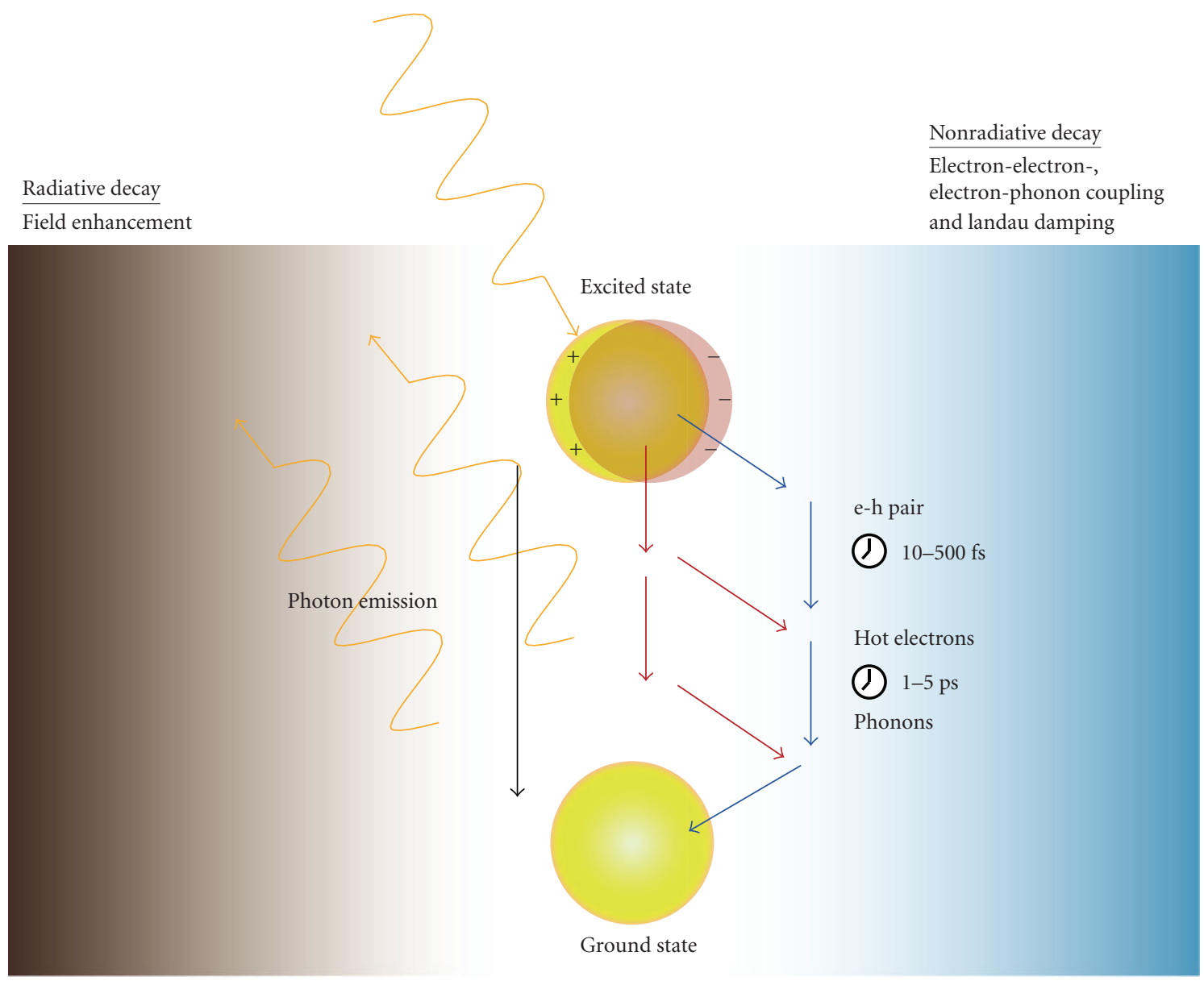

Figure 2: The principal decay paths for a localized (particle) plasmon excitation. On the left hand side, the radiative damping by photon emission and accompanying interferential effects. On the right hand side, the nonradiative damping by charge carrier- and direct heat generation. For details see [5].

bond formation $(0.5-6.5 \mathrm{eV})$. This should allow one to create conditions and propose schemes for enhanced (solar) light absorption in nanostructured materials and utilize the deposited energy to run chemical transformations such as water splitting. Figure 4, presenting previously unpublished results, shows that the extinction spectra of these structures are readily tuned via the metal particles' size.

In the case of plasmon-enhanced photochemistry, an area pioneered by Brus in the early eighties [41], both radiative and nonradiative enhancement pathways have been pursued, as illustrated in an extensive review article by Watanabe et al. [42]. To give two representative examples, the photocatalytic degradation of methylene blue was found to be significantly increased on nanocomposite photocatalysts consisting of silver nanoparticles embedded in titania. Radiative energy transfer from the Ag nanoparticles to the semiconductor was claimed to be the origin of increased efficiency $[43,44]$. Tian and Tatsuma, on the other hand, reported that ethanol and methanol can be photocatalytically oxidized by gold nanoparticle-nanoporous $\mathrm{TiO}_{2}$ composites (at the expense of oxygen reduction) under visible light illumination [45]. In this case, photoexcitation of the gold nanoparticles was followed by simultaneous charge transfer of electrons from the gold to the $\mathrm{TiO}_{2}$ conduction band and from a donor in solution to the gold nanoparticle.

The main idea behind our photodesorption study [8] was to investigate how the shape and size of silver clusters on a graphite surface will influence the interaction of light with adsorbate covered surface. Since the optical properties of the clusters are dominated by plasmon excitations, the photoinduced transformations will be indicative for their decay mechanisms. This was done by monitoring the changes in cross section for photodesorption of NO. NO was chosen as the probe molecule since its photoactivity on silver is well known. The photodesorption of NO from a silver substrate occurs via hot-electron scattering at the $2 \pi^{*}$ resonance of adsorbed NO with the hot carriers created in the substrate upon photon absorption (substrate-mediated hot electron driven process) $[46,47]$. Therefore, an experiment allowing comparison between the desorption cross sections in the cases of resonantly and nonresonantly excited nanoparticles will be indicative for the role of the plasmon particles and their energy dissipation.

The composition of the experimental model system was based on the following reasons. Silver clusters are known for their strong plasmonic response in the blue and near UV 


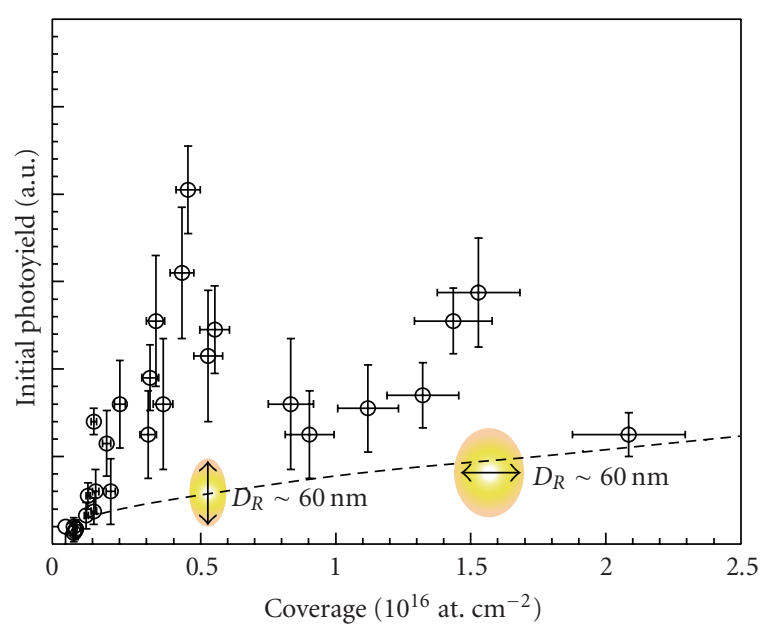

FIGURE 3: Elliptically-shaped silver nanoparticles show two separate plasmon resonance frequencies for light polarized parallel to the short and long axes, respectively. The graph demonstrates the effect of plasmon-mediated photodesorption of NO from Graphite surface (from [8]). The strong size dependent variation of the photoyield is in contrast with the smooth increase of the total Ag coverage (see dashed line).

part of the spectrum [5]. The plasmon resonance energy, when transferred to e-h pairs, will be sufficient to drive the bondbreaking of NO from the surface. From an experimental point of view, it is relatively simple to prepare silver clusters on graphite. Ag clusters on graphite grow in the VolmerWeber mode, resulting in well-defined individual particles with ellipsoidal shape [48].

The similar work functions of silver and graphite (4.5$4.8 \mathrm{eV}$ ) are also beneficial for our study since any changes in photodesorption cross sections will not be related to changes of work-function with increasing silver coverage.

Under pulsed irradiation with $355 \mathrm{~nm}$ photons, we observed a clear dependence of the initial photoyield on the average cluster size with a maximum yield at a silver coverage of $4.5 \cdot 10^{15}$ and $1.4 \cdot 10^{16}$ at. $/ \mathrm{cm}^{2}$ (Figure 3). Cluster size is dependent on surface coverage, and these amounts correspond to elliptical clusters with long, respectively short axis of ca. $60 \mathrm{~nm}$. The dashed line in Figure 3 shows that the expected signal increase as due to the larger surface area of the clusters. Obviously, the observations cannot be explained only by the higher cross-section $(\sim \times 6)$ from silver surface. We speculate that the enhancement of the total cross-section for NO photodesorption results from the enhanced optical absorption leading to increased hot charge carrier generation in graphite and the hot charge carrier generation in clusters.

4.2. Resonance Cavities and Waveguiding. Previously we have described how plasmonic particles may couple light into thin film waveguides. Here [49] we demonstrate how such coupling may be utilized to self-assemble periodic structures of the light scattering particles.

The experiments were done using suspended $100 \times$ $100 \mu \mathrm{m}^{2}$ wide and $40 \mathrm{~nm}$ thick membranes of $\mathrm{Si}_{3} \mathrm{~N}_{4}$, supported on the edges by bulk Si [50]. Upon the membranes,

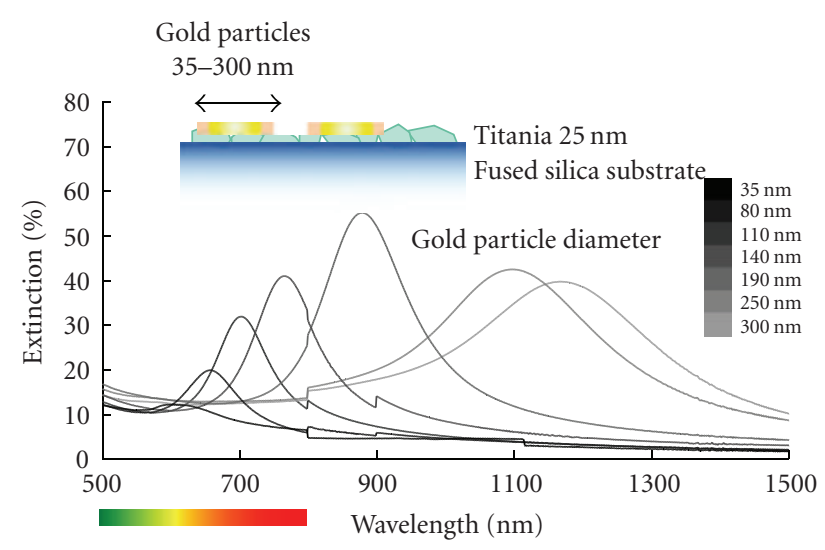

(a)

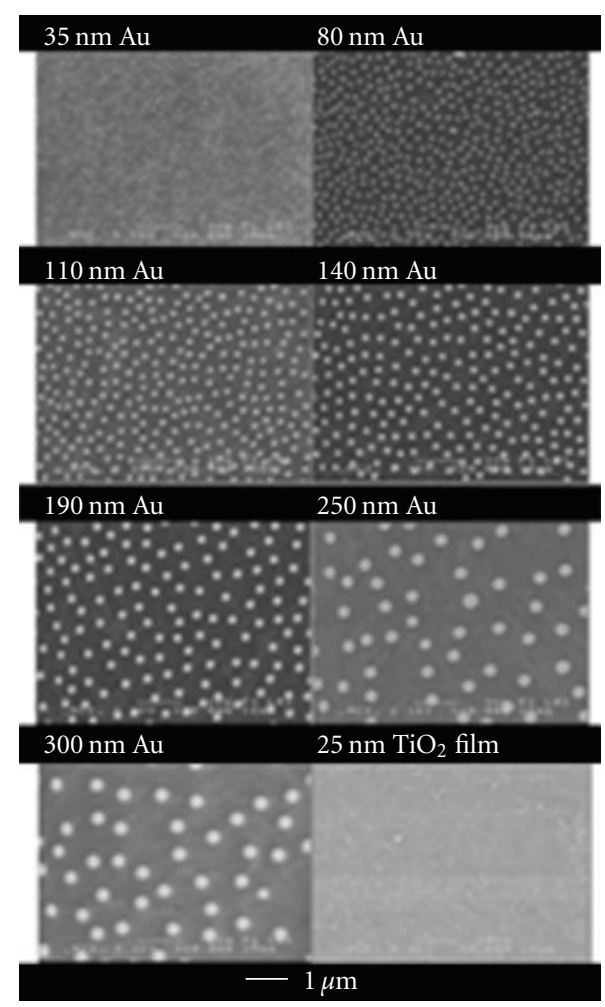

(b)

FIGURE 4: (a) Optical extinction spectra for gold nanoparticles with different diameters on a titania thin film. Inset is a schematic of the prepared nanoparticle photcatalysts. (b) SEM image of prepared films and their schematic composition.

a layer of gold, with thickness equivalent of $3 \mathrm{~nm}$, was thermally evaporated. Since gold, in this case, grows in the Volmer-Weber mode; the layer consisted of $15 \pm 12 \mathrm{~nm}$ sized islands.

When these structure were irradiated with single $5 \mathrm{~ns}$ laser pulses in the wavelength range of 532 to $640 \mathrm{~nm}$, gratings formed on the edge of the laser spot and on the edge of the $\mathrm{Si}_{3} \mathrm{~N}_{4}$ membrane. The latter grating formation occurred when the membrane edge and the laser spot coincided. A schematic picture of the grating formation 


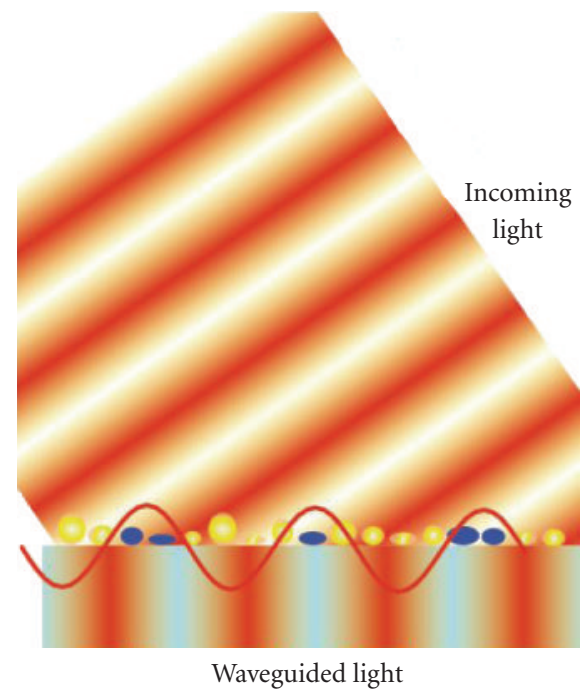

(a)

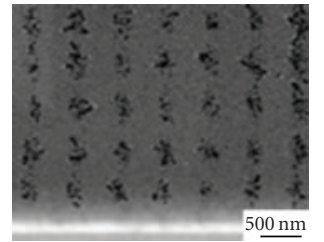

(b)

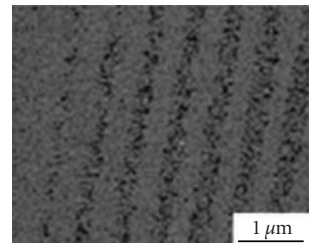

(c)

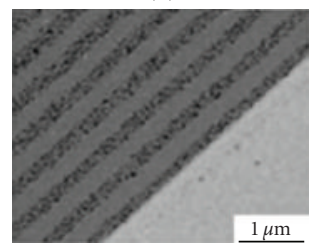

(d)

Figure 5: (a) Plasmonic particles scatter incoming coherent light into the underlying waveguide analogously with Figure 1(b). Due to stimulated emission of radiation from the particles into the waveguide, the guided light is coherent. Superposition of the incident light and the waveguided results in an amplitude modulated standing wave. Its time averaged intensity is here depicted as a sine curve. The plasmonic particles anneal (coalesce) at the time averaged intensity maxima of this modulated wave (blue particles) hereby forming the gratings. (b) Fringes formed at the intersection of the laser spot edge and the membrane edge. (c) Fringes formed on the laser spot edge. (d) Fringes formed on the membrane edge.

mechanism is presented in Figure 5(a) together with SEM micrographs of resulting gratings, from Figures $5(\mathrm{~b})$ to $5(\mathrm{~d})$.

The key mechanism behind the grating formation is the amplitude modulation of the field intensity on the metal surface arising when the waveguided field and the incident field superimpose. The time averaged energy density may be expressed according to

$$
\frac{E_{0}^{2}+E_{\mathrm{wg}}^{2}}{2}+E_{0} E_{\mathrm{wg}} \cos \left[\left(k_{0 x}-k_{\mathrm{wg}}\right) x+\varphi_{0}-\varphi_{\mathrm{wg}}\right] .
$$

Here $E_{0}$ and $E_{\mathrm{wg}}$ denote the amplitudes of the incident and waveguided electric fields, respectively, $k_{0 x}$ and $k_{\text {wg }}$ denote their wavevectors projected on the propagation direction of the guided wave, and finally, $\varphi_{0}$ and $\varphi_{\text {wg }}$ their phases. The periodicity of the fringes is hereby given by

$$
\frac{2 \pi}{\left|k_{0 x}-k_{\mathrm{wg}}\right|}
$$

This self-assembly of periodic gratings on a waveguide might become a useful tool in the fabrication of plasmonic light trapping devises in combination with particle shape control methods [51].

4.3. Geometrical Optical Resonance. Carbon materials are known for their very specific optical properties. For example, the relative high refractive index and moderate dispersion explain the brilliance of diamonds; the electron structure of amorphous carbon and graphite explains their strong absorption in the visible range [52]. The discovery of new carbon allotropes, the fullerenes, carbon nanotubes, and more recently graphene (single layers of graphite) [53-55] was noticed by directing most of the optical studies of nanosize carbon structures toward them. However, fabrication and exploration of the properties of nanostructures of the conventional carbon materials, glassy carbon (GC), and highly oriented pyrolytic graphite (HOPG) is of great interest per se and for comparison. Recently, we performed a systematic investigation of the correlation between optical absorption and the size of graphite nanostructures [56]. Samples with structure diameters ranging from 160 to $330 \mathrm{~nm}$ and heights from 60 to $190 \mathrm{~nm}$ were prepared.

In order to better understand the role and properties of carbon (graphite) substrate, we developed a process for fabrication of carbon nanostructures on the surfaces of highly oriented pyrolytic graphite (HOPG) and glassy carbon (GC) samples, using hole-mask colloidal lithography and oxygen reactive ion etching (RIE). Identical preparation schemes applied to the two materials yield structures with remarkably different shape and sizes. For example, $361 \mathrm{~nm}$ high and $37 \mathrm{~nm}$ diameter structures on glassy carbon compared to $120 \mathrm{~nm}$ high and $119 \mathrm{~nm}$ diameter structures on HOPG, see Figure 6(b). An important property of these structures, in the context of photo catalytic applications, is their strong optical absorption in the visible range. 


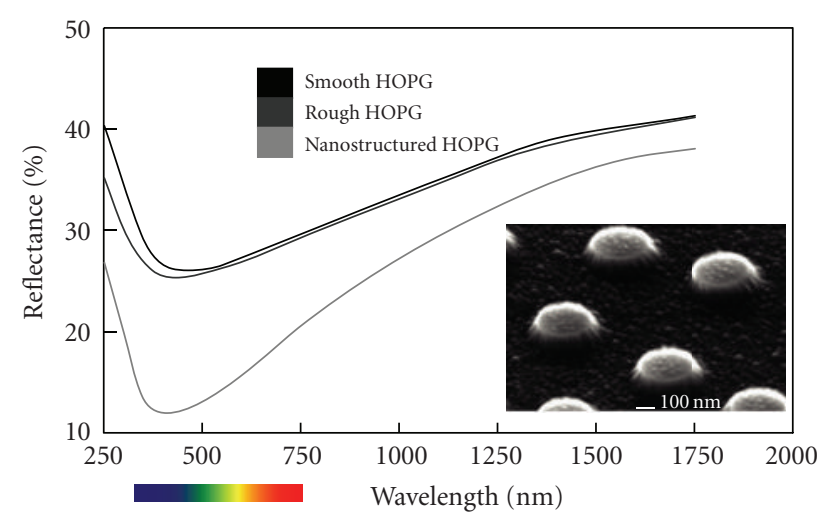

(a)

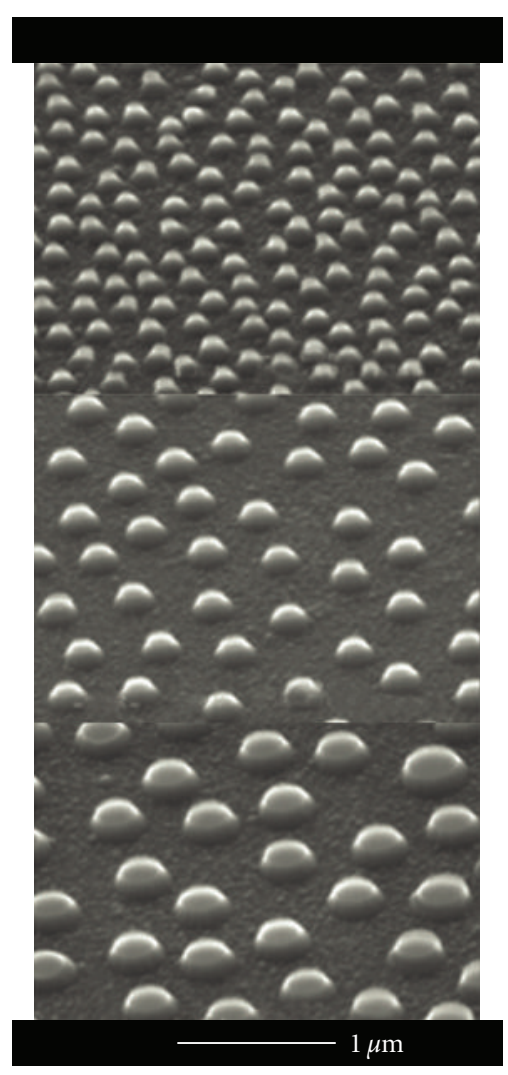

(b)

FIgURE 6: (a) Absorbance spectra recorded for different carbon structures. Nanostructured HOPG corresponds to inset micrograph. Rough HOPG corresponds to a surface like the one observed between the nanoparticles in the inset. (b) Graphite nanostructures fabricated on HOPG with HCL and reactive O-plasma etching.

The disk-like nanostructures were etched out of the basal plane surface of highly oriented pyrolytic graphite, using hole-mask colloidal lithography and oxygen reactive ion etching. Optical absorption spectra for wavelengths between 200 and $2500 \mathrm{~nm}$ were measured. Furthermore, electrodynamics calculations were conducted to model the optical properties of graphite nanostructures of similar sizes. Both the experimental and the theoretical work revealed

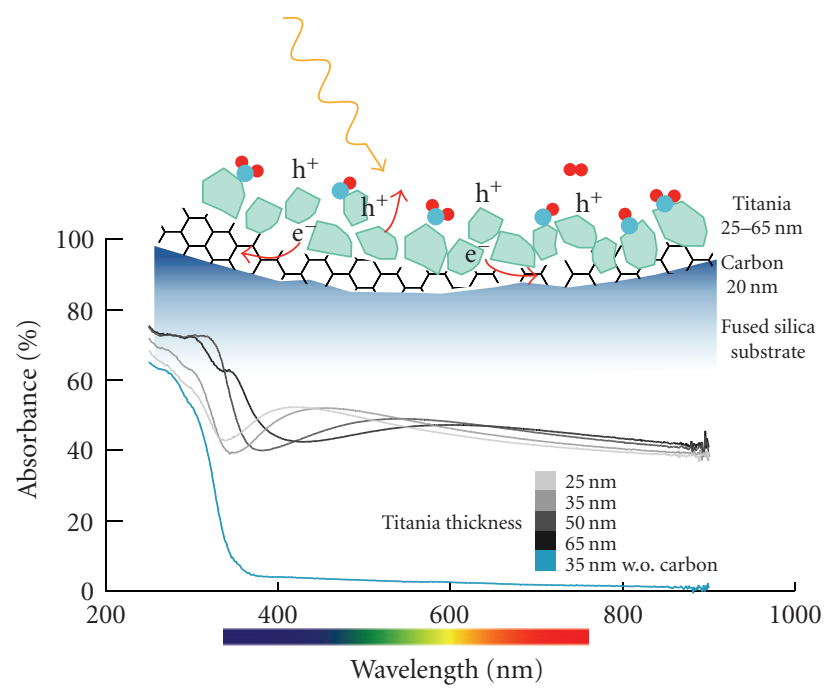

FIGURE 7: Optical absorption of $\mathrm{TiO}_{2} / \mathrm{C}$ composite films of different thicknesses compared to $35 \mathrm{~nm}$ thick pure titania film. Al films are deposited on fused silica substrate.

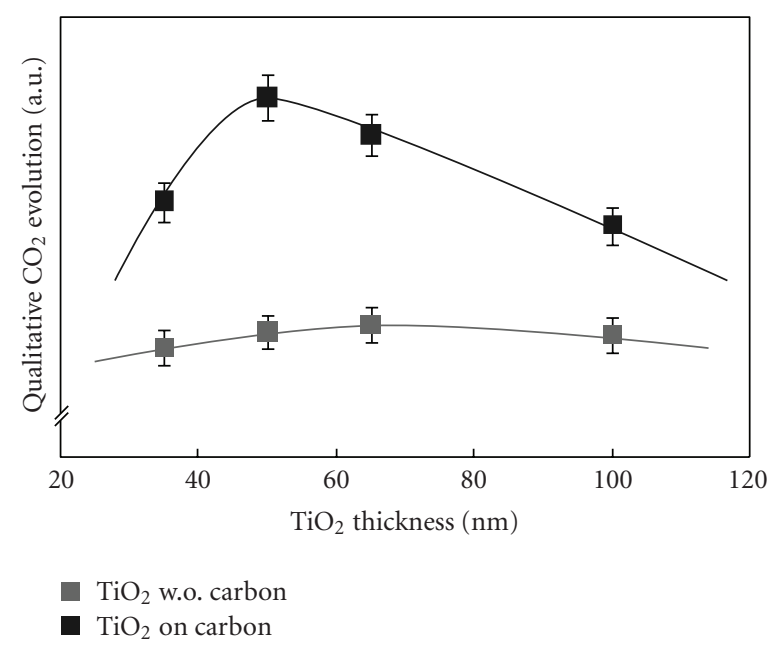

Figure 8: $\mathrm{CO}_{2}$ formation as result of photocatalytic oxidation of methanol under UV illumination. The results for composite films (dark data points) are compared with these for pure titania films (light data points) at several thickness. The solid lines are guides for the eye.

that resonant absorption correlated to the nanostructure diameters and heights. These absorption peaks are redshifted, from the visible for the smallest structures to near infrared for the largest. Simultaneously, the intensity of the absorption peaks increases for increasing structure heights, while increasing diameters results in decreased absorption.

4.4. Scattering Structures. Nanocrystalline $\mathrm{TiO}_{2}$ is widely used as photocatalyst due to its exceptional physiochemical properties. However, its wide bandgap $(\sim 3.2 \mathrm{eV})$ is the main restriction for it to be used for practical applications in solar energy conversion schemes. Similarly to many other studies [57], our aim is to improve the overall efficiency by enhanced 
solar light absorption in the visible regime. We work with $\mathrm{TiO}_{2}$ deposited on graphitic-like carbon film [58] as shown in Figure 7. Additionally to the enhanced absorption in the visible, the carbon film is expected to promote efficient separation of photogenerated charge carriers because of its anisotropic conductivity for electrons and holes $[59,60]$ As the photoreaction occurs on the surface of the $\mathrm{TiO}_{2}$ catalyst, it is of great interest to investigate thickness dependence of charge transport in the titania films and how the presence of carbon film affects the size of crystallites.

Both carbon and titania films were prepared by ebeam evaporation and DC reactive magnetron sputtering, respectively, at room temperature on a fused silica substrate. Postdeposition annealing was carried out to reduce internal stresses and improve crystallinity in the film at $800^{\circ} \mathrm{C}$ and $500^{\circ} \mathrm{C}$ for carbon and $\mathrm{TiO}_{2}$, respectively. Optical measurements of the composite films with different thicknesses are illustrated in Figure 7. The general observation is that composite films absorb more in the visible than pure titania films. Most importantly, the absorption threshold of $\mathrm{TiO}_{2}$ has red shifted for composite films.

The catalytic activity of the films was tested in the reaction of photo-oxidation of methanol to $\mathrm{CO}_{2}$ and water. The measurements were made in a, for this purpose constructed, minireactor $(\sim 880 \mu \mathrm{L}$ volume $)$ in batch mode under UV light illumination of $120 \mathrm{~mW} / \mathrm{cm}^{2}$. The products were monitored in situ by a mass spectrometer coupled to the reactor via a capillary. The rates of $\mathrm{CO}_{2}$ formation as a function of photocatalysts composition were obtained by monitoring $\mathrm{m} / \mathrm{z}=44$ mass spectrometer signal and are summarized in Figure 8. According to Figure 8, composite films clearly have enhanced photoactivity compared to pure titania films. The observed enhancement of photocatalytic activity is assigned to synergy effects at the carbon/ $/ \mathrm{TiO}_{2}$ interface, resulting in smaller titania crystallites and anisotropic charge carrier transport, which in turn reduces their recombination probability.

Variation in photoactivity with the thickness of the titania layer, both for pure and composite films, can be seen from the figure. The activity gradually increases up to certain thickness after which it starts to decrease. Once the film thickness reaches optimum value ( $\sim$ mean free path distance), it encounters more scattering on its way to the surface. As a consequence, the photoactivity decreases at thicknesses beyond the optimum due to enhanced trapping of charge carriers although the optical absorption volume of the films increases. Overall, this measurement clearly discriminates the effect of thickness on charge transport and photoactivity as discussed above.

\section{Conclusions}

With this paper, we have brushed the surface of what is possible to achieve in light harvesting with the aid of nanoscience and nanotechnology. Owing to miniaturized light trapping structures and enhanced absorption cross sections, solar devices may be designed with thicknesses well below the charge carrier diffusion lengths with maintained, or even altered, light harvesting efficiency.

\section{Acknowledgments}

Support by Swedish Foundation for Strategic Research (SSF, RAM08-0109) and Northen European Innovative Energy Research Programme (N-INNER, project \#30938Solar Hydrogen) is gratefully acknowledged.

\section{References}

[1] R. C. Willson and A. V. Mordvinov, "Secular total solar irradiance trend during solar cycles 21-23," Geophysical Research Letters, vol. 30, no. 5, 2003.

[2] M. B. McElroy, The Atmospheric Environment : Effects of Human Activity, Princeton University Press, Princeton, NJ, USA, 2002.

[3] J. T. Yates Jr., "Photochemistry on TiO2: mechanisms behind the surface chemistry," Surface Science, vol. 603, no. 10-12, pp. 1605-1612, 2009.

[4] M. Born and E. Wolf, Principles of Optics : Electromagnetic Theory of Propagation, Interference and Diffraction of Light, Cambridge University Press, New York, NY, USA, 7th edition, 1999.

[5] U. Kreibig and M. Vollmer, Optical Properties of Metal Clusters, Springer Series in Materials Science, Springer, New York, NY, USA, 1995.

[6] C. J. Wu and D. B. Wittry, "Investigation of minoritycarrier diffusion lengths by electron bombardment of Schottky barriers," Journal of Applied Physics, vol. 49, no. 5, pp. 28272836, 1978.

[7] H. J. Hovel, Solar Cells. Semiconductors an Semimetals, Academic Press, New York, NY, USA, 1975.

[8] K. Wettergren, B. Kasemo, and D. Chakarov, "Photodesorption of NO from graphite( $\left(\begin{array}{llll}0 & 0 & 0 & 1\end{array}\right)$ surface mediated by silver clusters," Surface Science, vol. 593, no. 1-3, pp. 235-241, 2005.

[9] P. Campbell and M. A. Green, "Light trapping properties of pyramidally textured surfaces," Journal of Applied Physics, vol. 62, no. 1, pp. 243-249, 1987.

[10] P. Campbell and M. A. Green, "High performance light trapping textures for monocrystalline silicon solar cells," Solar Energy Materials and Solar Cells, vol. 65, no. 1-4, pp. 369-375, 2001.

[11] C. Heine and R. H. Morf, "Submicrometer gratings for solarenergy applications," Applied Optics, vol. 34, no. 14, pp. 24762482, 1995.

[12] S. Ito, T. N. Murakami, P. Comte et al., "Fabrication of thin film dye sensitized solar cells with solar to electric power conversion efficiency over 10\%," Thin Solid Films, vol. 516, no. 14, pp. 4613-4619, 2008.

[13] L. I. Halaoui, N. M. Abrams, and T. E. Mallouk, "Increasing the conversion efficiency of dye-sensitized TiO photoelectrochemical cells by coupling to photonic crystals," The Journal of Physical Chemistry B, vol. 109, no. 13, pp. 6334-6342, 2005.

[14] P. Bermel, C. Luo, L. Zeng, L. C. Kimerling, and J. D. Joannopoulos, "Improving thin-film crystalline silicon solar cell efficiencies with photonic crystals," Optics Express, vol. 15, no. 25, pp. 16986-17000, 2007.

[15] K. J. Vahala, "Optical microcavities," Nature, vol. 424, no. 6950, pp. 839-846, 2003.

[16] J. B. Pendry, "Negative refraction makes a perfect lens," Physical Review Letters, vol. 85, no. 18, pp. 3966-3969, 2000.

[17] C. M. Soukoulis, M. Kafesaki, and E. N. Economou, "Negative-index materials: new frontiers in optics," Advanced Materials, vol. 18, no. 15, pp. 1941-1952, 2006. 
[18] A. P. Alivisatos, "Semiconductor clusters, nanocrystals, and quantum dots," Science, vol. 271, no. 5251, pp. 933-937, 1996.

[19] R. D. Schaller and V. I. Klimov, "High efficiency carrier multiplication in PbSe nanocrystals: implications for solar energy conversion," Physical Review Letters, vol. 92, no. 18, Article ID 186601, 4 pages, 2004.

[20] R. D. Schaller, M. Sykora, J. M. Pietryga, and V. I. Klimov, "Seven excitons at a cost of one: redefining the limits for conversion efficiency of photons into charge carriers," Nano Letters, vol. 6, no. 3, pp. 424-429, 2006.

[21] A. Shabaev, AL. L. Efros, and A. J. Nozik, "Multiexciton generation by a single photon in nanocrystals," Nano Letters, vol. 6, no. 12, pp. 2856-2863, 2006.

[22] I. Paci, J. C. Johnson, X. Chen et al., "Singlet fission for dye-sensitized solar cells: can a suitable sensitizer be found?" Journal of the American Chemical Society, vol. 128, no. 51, pp. 16546-16553, 2006.

[23] H. A. Atwater and A. Polman, "Plasmonics for improved photovoltaic devices," Nature Materials, vol. 9, no. 3, pp. 205213, 2010.

[24] H. R. Stuart and D. G. Hall, "Enhanced dipole-dipole interaction between elementary radiators near a surface," Physical Review Letters, vol. 80, no. 25, pp. 5663-5666, 1998.

[25] H. R. Stuart and D. G. Hall, "Island size effects in nanoparticleenhanced photodetectors," Applied Physics Letters, vol. 73, no. 26, pp. 3815-3817, 1998.

[26] F. U. J. Tsai, J. Y. Wang, J. J. Huang, Y. W. Kiang, and C. C. Yang, "Absorption enhancement of an amorphous Si solar cell through surface plasmon-induced scattering with metal nanoparticles," Optics Express, vol. 18, no. 13, pp. A207-A220, 2010.

[27] D. S. Wiersma, R. Sapienza, S. Mujumdar, M. Colocci, M. Ghulinyan, and D. L. Pavesi, "Optics of nanostructured dielectrics," Journal of Optics A: Pure and Applied Optics, vol. 7, no. 2, pp. S190-S197, 2005.

[28] B. Mayer and S. Madronich, "Actinic flux and photolysis in water droplets: mie calculations and geometrical optics limit," Atmospheric Chemistry and Physics, vol. 4, no. 8, pp. 22412250, 2004.

[29] V. E. Ferry, L. A. Sweatlock, D. Pacifici, and H. A. Atwater, "Plasmonic nanostructure design for efficient light coupling into solar cells," Nano Letters, vol. 8, no. 12, pp. 4391-4397, 2008.

[30] W. Bai, Q. Gan, F. Bartoli et al., "Design of plasmonic back structures for efficiency enhancement of thin-film amorphous Si solar cells," Optics Letters, vol. 34, no. 23, pp. 3725-3727, 2009.

[31] M. Kirkengen, J. Bergli, and Y. M. Galperin, "Direct generation of charge carriers in c-Si solar cells due to embedded nanoparticles," Journal of Applied Physics, vol. 102, no. 9, Article ID 093713, 2007.

[32] K. Kempa, J. Rybczynski, Z. Huang et al., "Carbon nanotubes as optical antennae," Advanced Materials, vol. 19, no. 3, pp. 421-426, 2007.

[33] P. V. Kamat, "Harvesting photons with carbon nanotubes," Nano Today, vol. 1, no. 4, pp. 20-27, 2006.

[34] G. Calzaferri, M. Pauchard, H. Maas, S. Huber, A. Khatyr, and T. Schaafsma, "Photonic antenna system for light harvesting, transport and trapping," Journal of Materials Chemistry, vol. 12, no. 1, pp. 1-13, 2002.

[35] V. P. Zhdanov and B. Kasemo, "Nanometer-sized antenna for enhancement of absorption of light by dye molecules," Applied Physics Letters, vol. 84, no. 10, pp. 1748-1749, 2004.
[36] D. M. Schaadt, B. Feng, and E. T. Yu, "Enhanced semiconductor optical absorption via surface plasmon excitation in metal nanoparticles," Applied Physics Letters, vol. 86, no. 6, Article ID 063106, pp. 1-3, 2005.

[37] M. Westphalen, U. Kreibig, J. Rostalski, H. Lüth, and D. Meissner, "Metal cluster enhanced organic solar cells," Solar Energy Materials and Solar Cells, vol. 61, no. 1, pp. 97-105, 2000.

[38] R. Corkish, M. A. Green, and T. Puzzer, "Solar energy collection by antennas," Solar Energy, vol. 73, no. 6, pp. 395401, 2002.

[39] C. Langhammer, B. Kasemo, and I. Zorić, "Absorption and scattering of light by $\mathrm{Pt}, \mathrm{Pd}, \mathrm{Ag}$, and Au nanodisks: absolute cross sections and branching ratios," The Journal of Chemical Physics, vol. 126, no. 19, Article ID 194702, 11 pages, 2007.

[40] J. W. Schwede, I. Bargatin, D. C. Riley et al., "Photon-enhanced thermionic emission for solar concentrator systems," Nature Materials, vol. 9, no. 9, pp. 762-767, 2010.

[41] L. Brus, "Noble metal nanocrystals: plasmon electron transfer photochemistry and single-molecule raman spectroscopy," Accounts of Chemical Research, vol. 41, no. 12, pp. 1742-1749, 2008.

[42] K. Watanabe, D. Menzel, N. Nilius, and H. J. Freund, "Photochemistry on metal nanoparticles," Chemical Reviews, vol. 106, no. 10, pp. 4301-4320, 2006.

[43] K. Awazu, M. Fujimaki, C. Rockstuhl et al., "A plasmonic photocatalyst consisting of silver nanoparticles embedded in titanium dioxide," Journal of the American Chemical Society, vol. 130, no. 5, pp. 1676-1680, 2008.

[44] P. Christopher, D. B. Ingram, and S. Linic, "Enhancing photochemical activity of semiconductor nanoparticles with optically active Ag nanostructures: photochemistry mediated by Ag surface plasmons," The Journal of Physical Chemistry C, vol. 114, no. 19, pp. 9173-9177, 2010.

[45] Y. Tian and T. Tatsuma, "Mechanisms and applications of plasmon-induced charge separation at $\mathrm{TiO} 2$ films loaded with gold nanoparticles," Journal of the American Chemical Society, vol. 127, no. 20, pp. 7632-7637, 2005.

[46] S. K. So, R. Franchy, and W. Ho, "Photodesorption of NO from $\operatorname{Ag}(111)$ and $\mathrm{Cu}(111)$," The Journal of Chemical Physics, vol. 95, no. 2, pp. 1385-1399, 1991.

[47] R. T. Kidd, D. Lennon, and S. R. Meech, "Comparative study of the primary photochemical mechanisms of nitric oxide and carbonyl sulfide on $\operatorname{Ag}(111)$," The Journal of Physical Chemistry B, vol. 103, no. 35, pp. 7480-7488, 1999.

[48] H. Hövel, B. Grimm, M. Pollmann, and B. Reihl, "Clustersubstrate interaction on a femtosecond time scale revealed by a high-resolution photoemission study of the fermi-level onset," Physical Review Letters, vol. 81, no. 21, pp. 4608-4611, 1998.

[49] L. Eurenius, C. Hägglund, E. Olsson, B. Kasemo, and D. Chakarov, "Grating formation by metal-nanoparticlemediated coupling of light into waveguided modes," Nature Photonics, vol. 2, no. 6, pp. 360-364, 2008.

[50] A. W. Grant, Q. H. Hu, and B. Kasemo, "Transmission electron microscopy 'windows' for nanofabricated structures," Nanotechnology, vol. 15, no. 9, pp. 1175-1181, 2004.

[51] R. Jin, Y. C. Cao, E. Hao, G. S. Métraux, G. C. Schatz, and C. A. Mirkin, "Controlling anisotropic nanoparticle growth through plasmon excitation," Nature, vol. 425, no. 6957, pp. 487-490, 2003.

[52] N. Laidani, R. Bartali, G. Gottardi, M. Anderle, and P. Cheyssac, "Optical absorption parameters of amorphous carbon films from Forouhi-Bloomer and Tauc-Lorentz models: a 
comparative study," Journal of Physics Condensed Matter, vol. 20, no. 1, Article ID 015216, 2008.

[53] A. K. Geim and K. S. Novoselov, "The rise of graphene," Nature Materials, vol. 6, no. 3, pp. 183-191, 2007.

[54] S. Iijima, "Helical microtubules of graphitic carbon," Nature, vol. 354 , no. 6348 , pp. 56-58, 1991.

[55] H. W. Kroto, J. R. Heath, S. C. O’Brien, R. F. Curl, and R. E. Smalley, “C60: buckminsterfullerene," Nature, vol. 318, no. 6042, pp. 162-163, 1985.

[56] H. Fredriksson, T. Pakizeh, M. Käll, B. Kasemo, and D. Chakarov, "Resonant optical absorption in graphite nanostructures," Journal of Optics A: Pure and Applied Optics, vol. 11, no. 11, Article ID 114022, 2009.

[57] A. L. Linsebigler, G. Lu, and J. T. Yates, "Photocatalysis on TiO2 surfaces: principles, mechanisms, and selected results," Chemical Reviews, vol. 95, no. 3, pp. 735-758, 1995.

[58] R. Sellappan and D. Chakarov, "Nanostructured $\mathrm{TiO}_{2} /$ Carbon films with enhanced photocatalytic activity for water dissociation," Journal of Molecular Catalysis A, vol. 335, no. 1-2, pp. 136-144, 2011.

[59] P. R. Wallace, “The band theory of graphite," Physical Review, vol. 71, no. 9, pp. 622-634, 1947.

[60] Y. Yao, G. Li, S. Ciston, R. M. Lueptow, and K. A. Gray, "Photoreactive TiO2/carbon nanotube composites: synthesis and reactivity," Environmental Science and Technology, vol. 42, no. 13, pp. 4952-4957, 2008. 


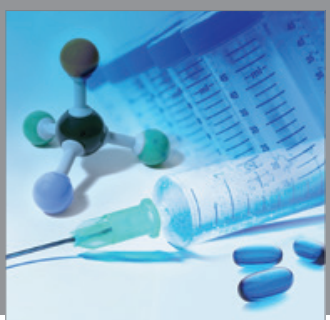

International Journal of

Medicinal Chemistry

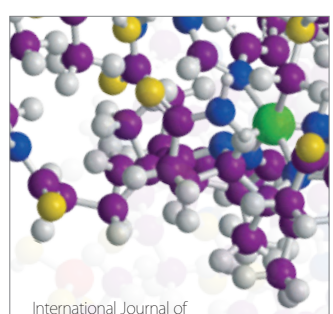

Carbohydrate Chemistry

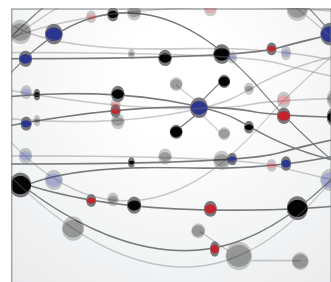

The Scientific World Journal
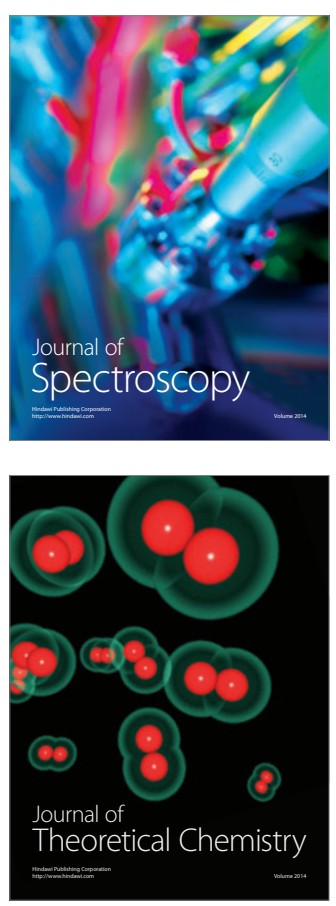
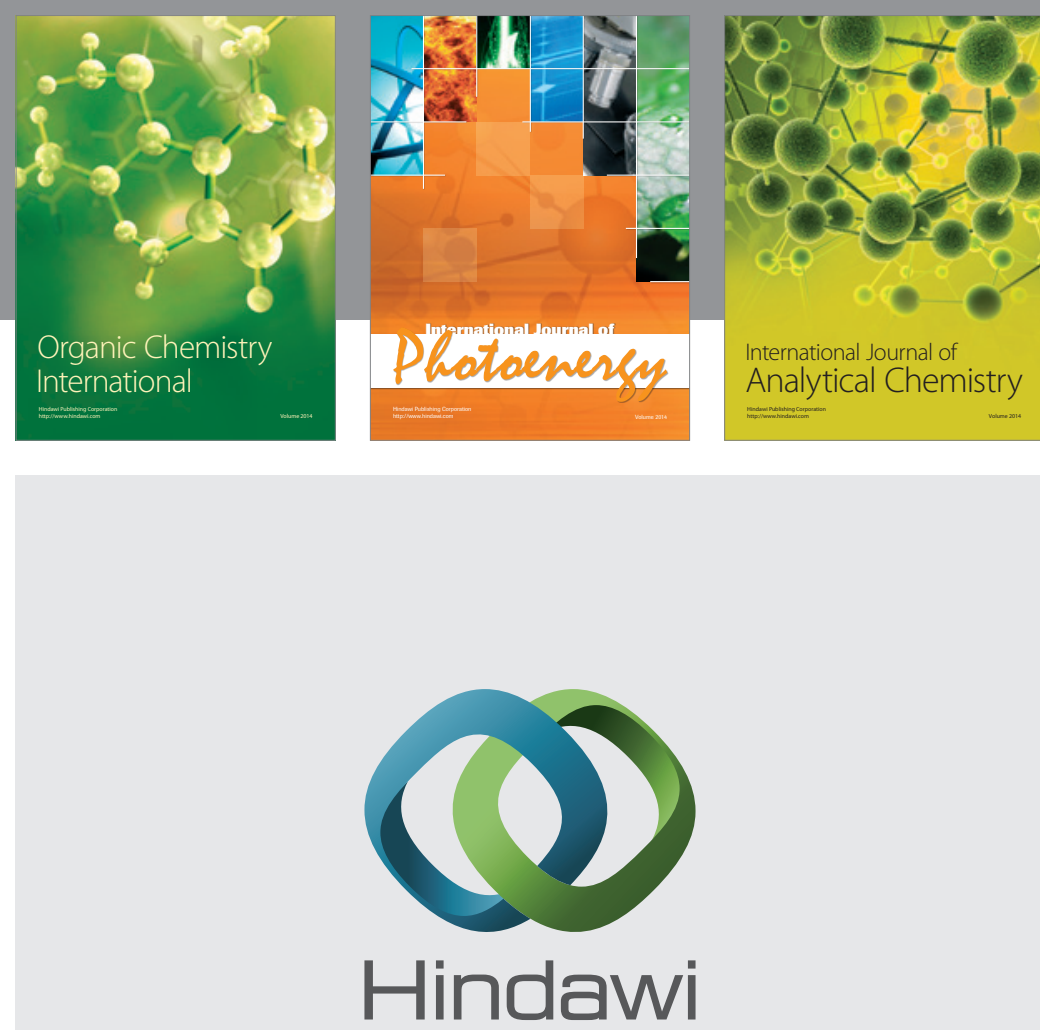

Submit your manuscripts at

http://www.hindawi.com
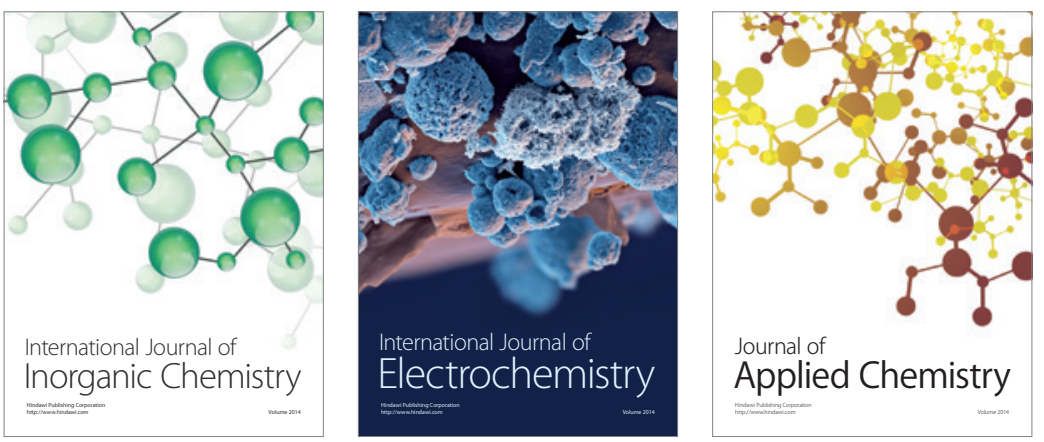

Journal of

Applied Chemistry
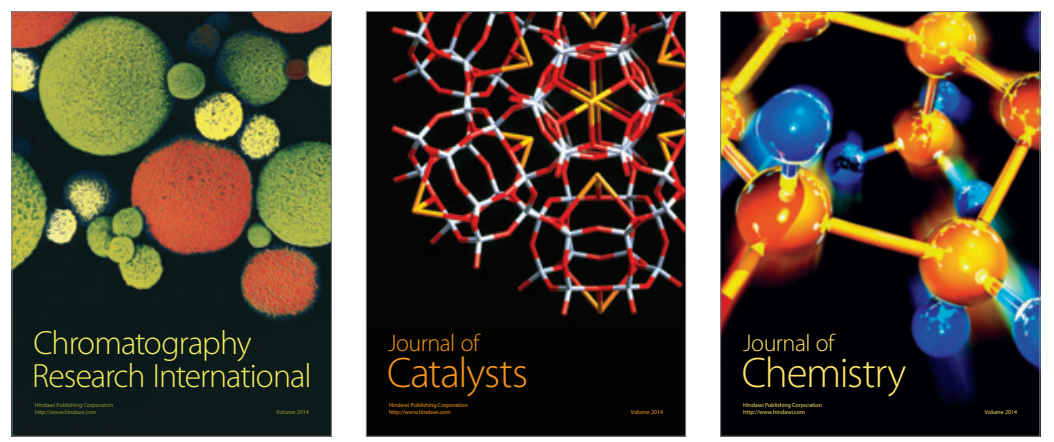
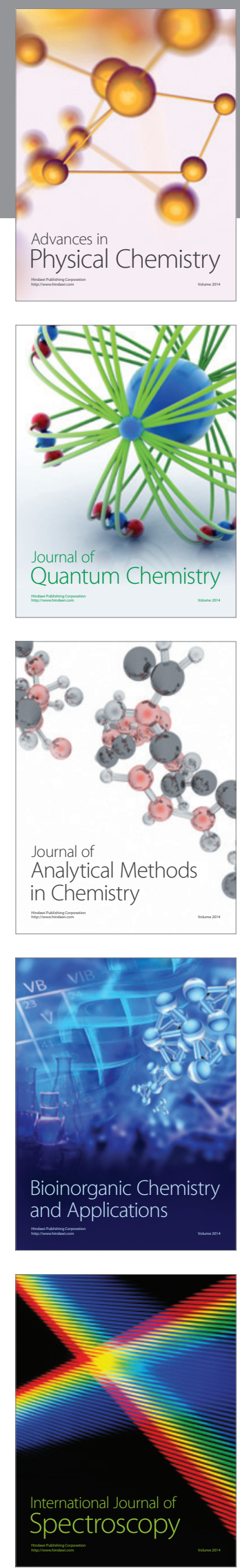